\title{
Efficient parallel spatial subdivision algorithm for object-based parallel ray tracing
}

\author{
Cevdet Aykanat, Veysi İşler and Bülent Özgüç
}

Parallel ray tracing of complex scenes on multicomputers requires the distribution of both computation and scene data to the processors. This is carried out during preprocessing and usually consumes too much time and memory. The paper presents an efficient parallel subdivision algorithm that decomposes a given scene into rectangular regions adaptively and maps the resultant regions to the node processors of a multicomputer. The proposed algorithm uses efficient data structures to identify the splitting planes quickly. Furthermore the mapping of the regions and the objects to the node processors is performed while parallel spatial subdivision proceeds. The proposed algorithm is implemented on an Intel iPSC/ 2 hypercube multicomputer and promising results have been obtained.

Keywords: ray tracing, spatial subdivision, multicomputers

In recent years, research on ray tracing has mostly concentrated on speeding up the algorithm by parallelization $^{1}$. There are two main approaches to the parallelization of ray tracing. One of them is image-space subdivision in which the computation related to various rays is distributed to the processors. The other approach is object-space subdivision, which should be adopted for the parallelization of ray tracing on distributed-memory message-passing architectures (multicomputers). Multicomputers are very promising architectures for massive parallelism because of their nice scalability features. In a multicomputer, there is no global memory, and synchronization and coordination between processors is achieved through message exchange. For an efficient parallelization on a multicomputer (called object-based parallel ray tracing), the object space data (the scene description with the auxiliary data structure) and computation should be distributed among processors of

Department of Computer Engineering and Information Science, Bilkent University, 06533 Ankara, Turkey

Paper received: 5 November 1993. Revised: June 1994 the multicomputer, since the whole object space data may not fit into the local memory of each processor for complex scenes.

The approach taken in this paper is to subdivide the 3D space containing the scene into disjoint rectangular subvolumes and assign both computation and the object data within a subvolume to a single processor. The proposed subdivision algorithm recursively bipartitions the rectangular subregions into two rectangular subsubregions starting from a given initial window until $P$ rectangular subregions are obtained, where $P$ denotes the total number of processors in the multicomputer. The subdivision and mapping should be performed in such a way that each processor is assigned an equal computational load. Furthermore, the neighbouring objects should be maintained in the local memories of adjacent node processors to achieve better data coherence ${ }^{1}$. The proposed subdivision algorithm also achieves the mapping of the rectangular subvolumes to processors during the decomposition process. The subdivision algorithm has efficient data structures to locate the splitting planes.

The spatial subdivision problem is a preprocessing overhead introduced for the efficient implementation of object-based parallel ray tracing on the target multicomputer. If the spatial subdivision algorithm is implemented sequentially, this preprocessing can be considered in the serial portion of the parallel ray tracing which limits the maximum parallel efficiency. For a fixed input scene instance, the execution times of the parallel ray tracing and the sequential subdivision programs are expected to decrease and increase, respectively, with an increasing number of processors in the target multicomputer. Thus, this preprocessing will begin to constitute a significant limit on the maximum efficiency of the overall parallelization owing to Amdahl's law. Hence, parallelization of the subdivision algorithm on the target multicomputer is a crucial issue for efficient object-based parallel ray tracing. In this work, we propose an efficient parallel spatial subdivision algorithm that utilizes the processors of the target multicomputer 
to be used for the object-based parallel ray tracing algorithm. After an initial random distribution of objects to processors, objects intermittently migrate during the execution of the recursive bisection algorithm in accordance with the mapping strategy such that all objects arrive at their home processors at the end of the parallel subdivision process. Each object traverses at most $\log _{2} P$ processors to reach its home processor.

\section{OBJECT-SPACE DECOMPOSITION}

The decomposition of object space data can be performed by utilizing the techniques that are developed to improve the naive ray tracing algorithm. These techniques are the hierarchy of bounding volumes ${ }^{2}$ and spatial subdivision ${ }^{3,4}$, and they can be adapted to parallel ray tracing as follows. The first technique forms a hierarchy of clusters consisting of neighbouring objects. In the parallel processing case, there might be two approaches, namely static and demand-driven, to accomplish a fair distribution of computation and storage. The former approach performs a static allocation by partitioning the entire hierarchy into a set of clusters, each of which is assigned to a node processor. This resembles a graph partitioning process ${ }^{5}$. The latter approach allocates object space data and relevant computation to the node processors on demand. The second technique, spatial subdivision, decomposes the $3 \mathrm{D}$ space containing the scene into disjoint rectangular prisms. As in the first technique, the resulting prisms are distributed to the node processors either statically or on demand ${ }^{1,5-7}$.

In this paper, the second technique, spatial subdivision, is used to decompose the object space data. Spatial subdivision can be performed in several ways that give rise to different rectangular volumes. Regular subdivision $^{8}$, octrees ${ }^{3}$ and binary space partitioning (BSP) $)^{4,9}$ are widely used spatial subdivision schemes.

\section{Utilizing BSP in parallel ray tracing}

Although both octree and regular subdivision schemes have very useful properties when used with a conventional ray tracing algorithm, it is difficult to achieve a computational load balance among processors if some coherence properties, such as object, data, and image coherence, are to be utilized. A manifestation of coherence called data coherence, first exploited by Green and Paddon ${ }^{1}$, is a very powerful and useful property that may reduce the communications overhead. Communications among the node processors is one of the most time consuming operations in an object-based parallel ray tracing system. Therefore, exploiting data coherence is essential in speeding up object-based parallel ray tracing. In order to exploit data coherence, we propose a variant of BSP which we call balanced binary space partitioning (BBSP), since a complete binary tree is generated at the end of the subdivision. The subdivision is carried out for a window defined over a viewing plane onto which the objects in the scene are projected (in parallel) (see Figure 1). The subdivision produces a set of rectangular regions on the window, and a set of $3 \mathrm{D}$ volumes is obtained by extending the rectangular regions in the viewing direction. By means of this subdivision preprocess, the decomposition of both object space data describing the

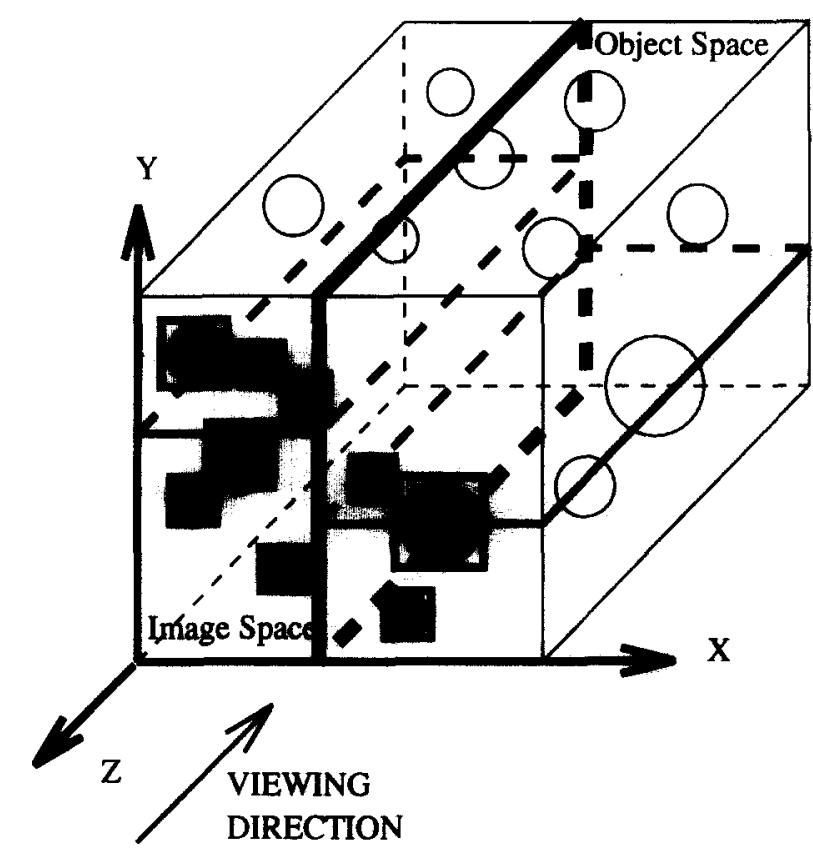

Figure 1 4-way subdivision of scene using BBSP

scene and the image-space computation associated with the pixels on the window are performed. It is assumed that the viewing volume and the $3 \mathrm{D}$ volumes produced have a rectangular (parallepiped) shape rather than a pyramid shape.

The proposed BBSP algorithm starts by projecting all the objects in the view volume onto a given window $W$. The window $W$ is the initial rectangular region for the recursive subdivision process. In the following steps of the algorithm, each rectangular subregion generated is subdivided into two subsubregions by a splitting plane which is parallel to either the $x z$ (horizontal) or $y z$ (vertical) plane as shown in Figure 1. This recursive subdivision process proceeds in a breadth-first manner until the number of generated subregions (at the leaves of the recursion tree) becomes equal to the number of procesors. Here, the number of processors is assumed to be a power of 2 .

The proposed algorithm decomposes both the image space and the object space, and meanwhile maps the resulting image-space subregions and the respective object-space subvolumes to the processors in one phase. Each 3D subvolume is labelled using the label of the respective $2 \mathrm{D}$ subregion from which the $3 \mathrm{D}$ volume has been obtained. Each 3D volume and its corresponding $2 \mathrm{D}$ region are then assigned to the node processor that has a node number that is equal to the label of the volume. Identifying the position of the splitting plane (i.e. subdivision) and labelling the generated regions (i.e. mapping) are key operations in the algorithm.

\section{Identifying optimal splitting planes}

The subdivision is practically carried out on the screen since the window is mapped to the viewport that is defined on a display device (screen). A splitting plane thus divides a given rectangular region of the screen into two disjoint rectangular subregions consisting of pixels. A rectangular region on the screen can be subdivided into two using either a horizontal splitting plane or a vertical splitting plane. Either a vertical or a horizontal splitting plane with minimum cost is chosen from all the possible 
vertical and horizontal splitting planes on the basis of an objective (cost) function. Hence, a splitting plane is characterized by its cost, direction (vertical or horizontal) and its location where the screen is cut.

In BSP trees, the location of the splitting plane is usually chosen along either the object median or the spatial median. MacDonald and Booth ${ }^{10}$ have examined two heuristics for space subdivision using BSP. They pointed out that the probability of the intersection of a given ray with an object is proportional to the surface area of the object; this is called the surface area heuristic. Using this heuristic, they have also found out that the optimal splitting plane lies between the object median and the spatial median. This result reduces the search range required to find out the location of the splitting plane. However, it is still an expensive operation to search within the reduced search range. Furthermore, the analysis in Reference 10 neglects the existence of shared objects between the generated subregions.

In this work, we propose an efficient search algorithm for identifying optimal splitting planes during recursive space subdivision. The proposed search algorithm uses efficient data structures and requires only integer arithmetic. In the proposed algorithm, the position of an optimal splitting plane is determined by using an objective function that considers both the minimization of the computational load imbalance and the number of objects shared between the generated subregions. The proposed objective function exploits the surface area heuristic to maintain the computational load balance between the generated subregions.

\section{Objective function}

The cost of a vertical splitting plane $b$ on a window $W$ consisting of $n \times m$ pixels (resolution) is defined as

$$
C_{\mathrm{v}}(b)=\frac{\left|n \times b \times L_{b}-n \times(m-b) \times R_{b}\right|}{n \times m \times N}+\frac{S_{b}}{N}
$$

for $b=1, \ldots, m$, where $N$ denotes the total number of objects projected onto the window $W$ under consideration. The objective function for a horizontal splitting plane can easily be obtained by exchanging $n$ with $m$ in Equation 1. Here, $L_{b}$ and $R_{b}$ denote the number of objects on the left (below) and right (above) of the vertical (horizontal) splitting plane $b$, respectively. Furthermore, $S_{b}$ denotes the number of shared objects straddling the splitting plane $b$.

The denominator of the first term in Equation 1 denotes the total computational load associated with the window when only primary rays are considered. Hence, the first term in Equation 1 represents the percentage load imbalance between the two subregions generated by a particular splitting plane. Similarly, the second term in Equation 1 denotes the percentage of objects shared between these two subregions. The shared objects cause several problems. First, the shared objects are duplicated in the local memories of the processors to which these objects are assigned. Second, an intersection test with a shared object might be repeated if the first intersection point was not inside the subvolume that was assigned to the processor performing the test. As in conventional ray tracing, there might be another closer intersection point within the next subvolume along the path of the ray.

The objective function in Equation 1 is computed for all the splitting planes in both the vertical and horizontal directions. The splitting plane with the smallest cost is chosen as the optimal splitting plane. Hence, the objective function should be efficiently computed. The objective function for vertical splitting planes can be simplified as

$$
C_{\mathrm{v}}(b)=\frac{1}{m \times N}\left\{\left|b \times L_{b}-(m-b) \times R_{b}\right|+m \times S_{b}\right\}
$$

for $b=1, \ldots, m$. The simplification for horizontal splitting planes can be obtained by replacing $m$ with $n$ in Equation 2. The parameter $1 / N$ can be neglected since it is a constant factor common in all cost computations (both vertical and horizontal). Similarly, the parameters $1 / m$ and $1 / n$ appear as constant factors that are common in vertical and horizontal splitting plane computation, respectively. Hence, it is sufficient to compute the following functions:

$$
\begin{aligned}
& C_{\mathrm{v}}(b)=\left|\left\{b \times L_{b}+(m-b) \times R_{b}\right\}\right|+m \times S_{b} \\
& C_{\mathrm{h}}(b)=\left|\left\{b \times L_{b}+(n-b) \times R_{b}\right\}\right|+n \times S_{b}
\end{aligned}
$$

for $b=1, \ldots, m$ and $b=1, \ldots, n$, in order to find the optimal vertical and horizontal splitting planes $b_{v}^{\text {min }}$ and $b_{\mathbf{h}}^{\min }$, respectively. The optimal splitting plane is then chosen from these two splitting planes by comparing $C_{\mathrm{v}}\left(b_{\mathrm{v}}^{\mathrm{min}}\right) / m$ with $C_{\mathrm{h}}\left(b_{\mathrm{h}}^{\min }\right) / n$. This formulation enables only integer arithmetic to be used during the cost computation.

\section{Data structures}

Horizontal and vertical splitting planes subdivide a given rectangular region in $2 \mathrm{D}$ and $1 \mathrm{D}$, respectively. Two integer arrays are defined to hold the information related to the distribution of objects along each one of these two dimensions. To form the data structures, the objects are projected onto the viewing plane and the projections of the objects are surrounded by bounding boxes to simplify the computation as seen in Figure 2. After this operation, each object $o$ in the scene has four attributes: $x \min [o], x \max [o], y \min [o]$ and $y \max [o]$. Here, $x \min [o](y \min [o])$ and $x \max [o](y \max [o])$ denote the left (bottom) and the right (top) borders of the bounding box of an object $o$, respectively. Assuming that the window $W$ consists of $n \times m$ pixels (resolution), the arrays for $x$ and $y$ dimensions have a size of $m$ and $n$, respectively. The following major data structures are constructed and used for the $x$ dimension: $X$ MinCntr and $X$ MaxCntr. $X$ MinCntr $[b]$ and $X$ MaxCntr $[b]$ contain the number of objects whose $x \min$ and $x \max$ values are equal to $b$, respectively, for $b=1,2, \ldots, m$. $Y$ MinCntr and YMaxCntr are similar data structures constructed and used for the $y$ dimension.

Having formed these data structures, a prefix sum operation is performed on these integer arrays. These integer arrays are then used in the computation of the objective functions in Equations 3 and 4. These equations need the values of $R_{b}, L_{b}$ and $S_{b}$ for each possible splitting position $b$. After prefix sum operations, $X$ MinCntr $[b]$ and $X$ MaxCntr[b] contain the number of objects whose $x \min$ and $x \max$ values are equal to or less than $b$, respectively. Hence, $X \mathrm{MinCntr}[b](Y M i n C n t r[b])$ denotes the number $L_{b}$ of objects in the left (bottom) subregion of the vertical (horizontal) splitting plane. Similarly, $X$ MaxCntr[b] (YMaxCntr[b]) denotes the number of 


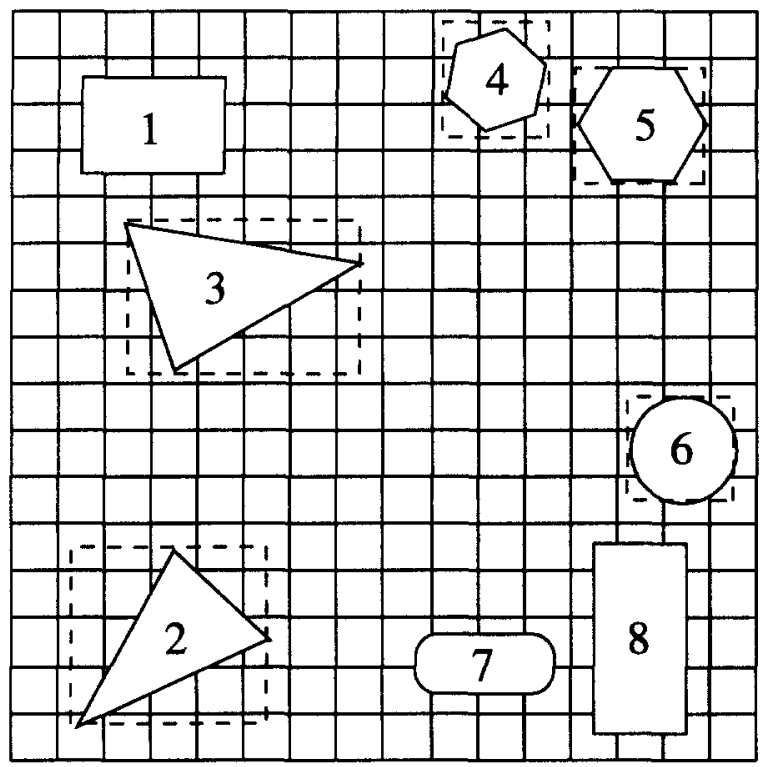

XMinCntr

\begin{tabular}{|l|l|l|l|l|l|l|l|l|l|l|l|l|l|l|l|}
\hline 0 & 2 & 3 & 3 & 3 & 3 & 3 & 3 & 4 & 5 & 5 & 5 & 7 & 8 & 8 & 8 \\
\hline
\end{tabular}

XMaxCntr

\begin{tabular}{|l|l|l|l|l|l|l|l|l|l|l|l|l|l|l|l|}
\hline 0 & 0 & 0 & 0 & 1 & 2 & 2 & 3 & 3 & 3 & 3 & 5 & 5 & 5 & 7 & 8 \\
\hline
\end{tabular}

Figure 2 Sample scene projected onto viewing plane [XMinCntr and XMaxCntr contain values after the prefix sum operation.]

objects in the left (bottom) subregion which do not straddle the vertical (horizontal) splitting plane $b$. Hence, $S_{b}$ and $R_{b}$ can easily be computed as

$$
\begin{aligned}
& S_{b}=L_{b}-X \operatorname{MaxCntr}[b] \\
& R_{b}=\left(N+S_{b}\right)-L_{b}
\end{aligned}
$$

for a vertical splitting plane $b$. For a horizontal splitting plane $b, S_{b}$ and $R_{b}$ can similarly be computed using these two equations by replacing $X$ MaxCntr in Equation 5 with $Y$ MaxCntr. Note that the values of $R_{b}, L_{b}$ and $S_{b}$ are efficiently computed using only three integer additions which will be performed for all possible splitting planes.

\section{Mapping}

The proposed algorithm carries out the mapping of the generated subregions during the recursive subdivision process. Each generated subregion is assigned a label that corresponds to the processor group to which it is assigned. Initially, the window $W$ is assumed to be assigned to all the processors in the parallel architecture. While splitting a region into two subregions, the processor group assigned to that region is also split into two halves, and these two halves are assigned those two subregions, respectively. This recursive spatial subdivision of the window proceeds together with the recursive subdivision of the processor interconnection topology. The recursive subdivision and assignment scheme to be adopted for the processor interconnection topology is a crucial factor in achieving the data coherence mentioned above.

In this work, we propose a recursive labelling scheme for the regions generated during the recursive subdivision of the window. This labelling scheme emulates the recursive definition of the hypercube interconnection topology as the target architecture for the object-based parallel ray tracing algorithm. However, the proposed labelling can easily be adapted to other parallel architectures implementing symmetric and recursive interconnection topologies (e.g. 2D mesh and 3D mesh) with minor modifications.

Here, we briefly summarize the topological properties of hypercubes exploited in the proposed labelling. A multicomputer implementing the hypercube interconnection topology consists of $P=2^{d}$ processors with each processor being directly connected to $d$ other neighbour processors. In a $d$-dimensional hypercube, each processor can be labelled with a $d$ bit binary number such that the binary label of each processor differs from its neighbour by exactly 1 bit. A channel $c$ defines the set of $P / 2$ links connecting neighbour processors whose binary labels differ only by bit $c$, for $c=0,1,2, \ldots, d-1$. In the recursive definition of the hypercube topology, a $d$-dimensional hypercube is constructed by connecting the processors of two $(d-1)$-dimensional hypercubes in a one-to-one manner. Hence, a $d$-dimensional hypercube can be subdivided into two disjoint $(d-1)$-dimensional hypercubes, called subcubes, by tearing the hypercube across a particular channel (e.g. $c=d-1$ ). Each one of these two $(d-1)$-dimensional subcubes can in turn be divided into two disjoint $(d-2)$-dimensional subcubes by tearing them across another channel (e.g. $c=d-2$ ). Hence, $d$ such successive tearings along different channels (e.g. $c=d-1$, $d-2, \ldots, 1,0)$ result in $2^{d} 0$-dimensional subcubes (i.e. processors). An $h$-dimensional subcube in a $d$-dimensional hypercube $(0 \leqslant h \leqslant d)$ can be represented by a $d$-tuplet containing $h$ free coordinates $(x \mathrm{~s})$ and $d-h$ fixed coordinates $(0 \mathrm{~s} \text { and } 1 \mathrm{~s})^{1{ }^{1}}$.

In the proposed mapping scheme, the label $Q$ of the initial rectangular region (window $W$ ) is initialized to null. Consider the subdivision of a particular subregion labelled as $Q$ by a vertical or horizontal splitting plane. Note that the label $Q$ of this subregion is a $q$ bit binary number, where $q$ denotes the depth of this subregion in the subdivision recursion tree. Hence, subregion $Q$ is already mapped to the $(d-q)$-dimensional subcube $Q x \ldots x$. The left (below) and right (above) subsubregions generated by a vertical (horizontal) splitting plane are labelled as $Q 0$ and $Q 1$, respectively. This labelling corresponds to tearing the subcube $Q x \ldots x$ across channel $d-q-1$ and mapping the resulting $(d-q-1)$ dimensional subsubcubes $Q 0 x \ldots x$ and $Q 1 x \ldots x$ to left (below) and right (above) subsubregions, respectively (see Figure 3). However, if two subregions $Q 0$ and $Q 1$ generated from the same region by a vertical (horizontal) splitting plane are both split again by vertical (horizontal) planes, then the subsubcube-to-subsubregion assignment in one of these two subregions is performed in reverse order. The proposed labelling scheme tries to maximize the data coherence by mapping neighbouring subregions to neighbouring subcubes, to as great a degree as possible, during the recursive subdivision process. Figure 4 illustrates the possible labelling combinations in a particular subpath of the recursion tree.

\section{PARALLEL SPATIAL SUBDIVISION}

For complex scenes, spatial subdivision using the proposed BBSP scheme may still take too much time. 


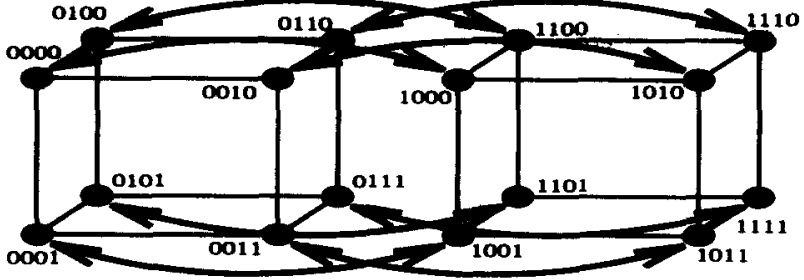

Subdivision across channel $\mathrm{c}=3$

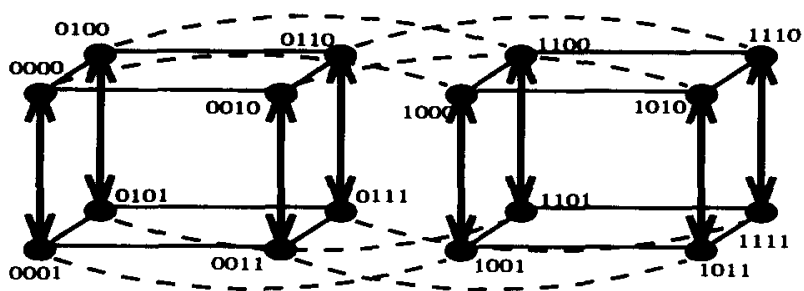

Subdivision across channel $c=2$

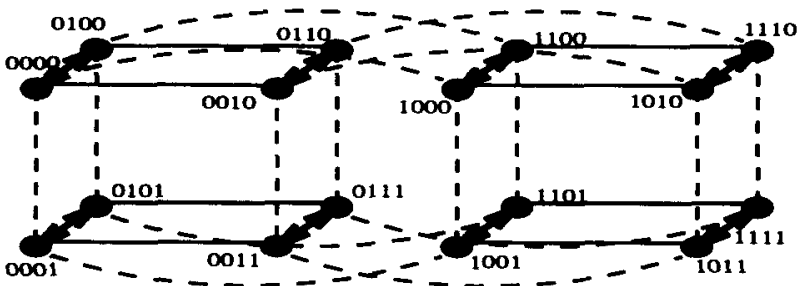

Subdivision across channel $\mathrm{c}=1$

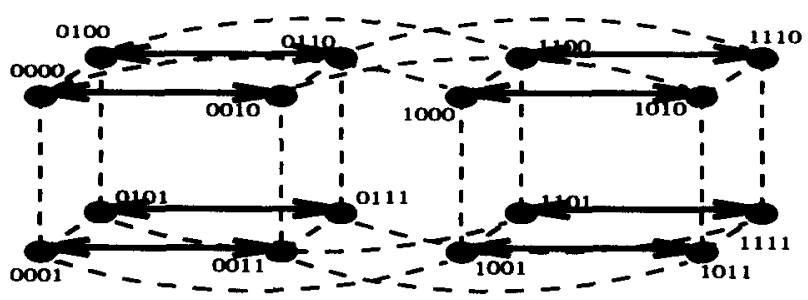

Subdivision across channel $\mathrm{c}=0$

Figure 3 Operation structure of proposed parallel BBSP algorithm

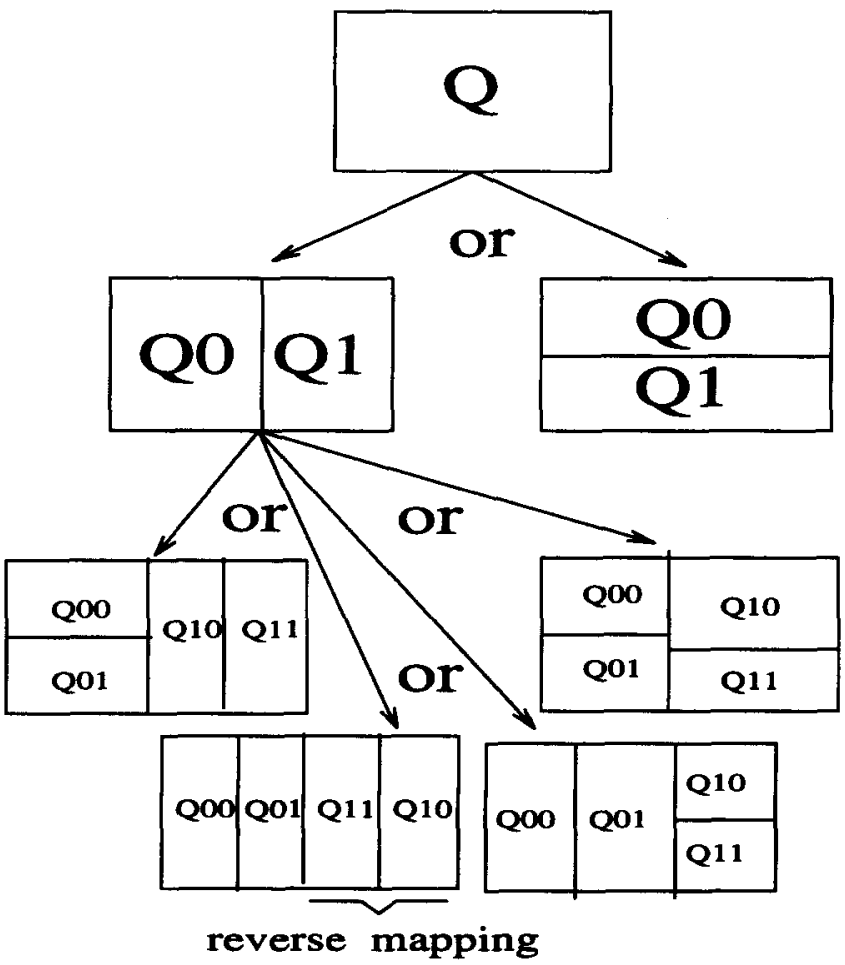

Figure 4 Subregion labelling for sample case
We may therefore use the node processors of the target multicomputer to speed up the subdivision process. Furthermore, these processors are already idle waiting for the start of the ray-tracing loop. This approach increases the utilization of the parallel system. Reduction of the spatial subdivision time has also been studied by other researchers. McNeill et al. ${ }^{12}$ have suggested an algorithm for dynamic building of the octree to reduce the data structure generation time. In this work, we propose a parallel subdivision algorithm, a parallel version of the BBSP scheme for hypercube multicomputers. The proposed BBSP algorithm is based on the divide-and-conquer paradigm. Hence, the BBSP algorithm is very suitable for parallelization on hypercubes because of their recursive structures, mentioned above. The proposed parallel BBSP algorithm has a very regular communications structure and it requires only concurrent single-hop communications (i.e. communications between neighbour processors) on hypercubes. The proposed parallel BBSP algorithm may also be adapted to other interconnection topologies. However, multihop communications may be required in other topologies.

In the proposed scheme, the host processor randomly decomposes the object database into $P$ even subsets such that each subset contains either $\lceil N / P\rceil$ or $\lfloor N / P\rfloor$ objects, and it sends each subset to a different node processor of the hypercube. Then, the following steps are performed in a divide-and-conquer manner ( $d=\log _{2} P$ times) for each channel $c$ from $c=d-1$ down to $c=0$.

(1) Node processors concurrently construct their local integer arrays corresponding to their local object database.

(2) Processors concurrently perform a prefix-sum operation on their local integer arrays.

(3) Processors of each $(c+1)$-dimensional disjoint subcube perform a global vector sum operation on their local integer arrays. Note that $2^{d-c-1}$ global vector-sum operations are performed concurrently. At the end of this step, processors of each $(c+1)$-dimensional subcube will accumulate the same local copies of the prefix-summed integer arrays.

(4) Replicated integer arrays on the $x$ and $y$ dimensions in each subcube are virtually divided into $2^{c+1}$ even slices, and each slice is assigned to a different processor of that subcube. Then, processors perform the cost computation of the splitting planes corresponding to their slices in order to find their local optimum splitting planes.

(5) Processors of each subcube perform a global minimum operation to locate the optimal splitting plane corresponding to the subregion mapped to that subcube.

(6) Processors of each subcube determine their local subsubregion assignment for the following stage $c-1$, according to the proposed mapping scheme. Then, processors concurrently perform a single pass over their local object database to gather and send the objects which belong to the other subsubregion to their neighbours on channel $c$. Hence, two subsubcubes of each 
subcube effectively exchange their subset of local object databases such that each subsubcube collects the object database corresponding to its subsubregion assignment in the following stage $c-1$. Note that $2^{d-c-1}$ subsubcube pairs perform this exchange operation concurrently.

During Step 6, processor pairs also determine their local shared objects which are not involved in the exchange operation. However, processors update either $x \mathrm{~min}$ ( $y$ min) or $x \max (y \max )$ values of their local shared objects according to their subsubregion assignment for a vertical (horizontal) splitting plane. Hence, processors maintain and process disjoint rectangular parts of the bounding boxes corresponding to the shared objects.

Figure 3 illustrates the operation structure of the proposed parallel BBSP algorithm on a 4D hypercube topology. In Figure 3, links drawn as broken lines illustrate the idle links in a particular stage of the parallel algorithm. Links drawn as solid lines illustrate the disjoint subcubes working concurrently and independently for the subdivision of their subregions at each stage. That is, processors of each subcube work in cooperation to determine the optimal subdivision of the subregion assigned to that subcube. These links also show the subcubes in which intrasubcube global vector-sum and global minimum operations are performed. In Figure 3, links drawn as solid lines with arrows illustrate the channel over which the object-exchange operation takes place. These links also illustrate the subdivision of each subcube into two disjoint subsubcubes at the end of each stage. As is also seen in Figure 3, all the objects arrive at their home processors after $\log _{2} P$ concurrent object-exchange operations. Note that shared objects will have more than one home processor, and they will be replicated in those processors.

\section{EXPERIMENTAL RESULTS}

The proposed parallel subdivision algorithm is implemented on an Intel iPSC/ 2 hypercube multicomputer with 16 processors. The performance of the parallel program is tested on several scenes containing different numbers of objects.

As mentioned above, the computational load balance and communications overheads are two crucial factors that determine the efficiency of a parallel algorithm. The recursive spatial bisection scheme used in the BBSP algorithm tries to maintain a load balance among the disjoint $(c+1)$-dimensional subcubes at each subdivision stage $c$ during the first level of the parallel ray tracing computation. That is, in a particular subdivision stage $c$, the products of the numbers of local objects and areas of the rectangular subregions assigned to disjoint subcubes are approximately equal to each other. Note that, at the end of each stage of the parallel subdivision algorithm (Step 6), objects always migrate to their destination subcubes for the following stage. That is, at the beginning of each subdivision stage, each subcube holds only the local objects which belong to its respective local rectangular subregion. However, in the subdivision algorithm, the complexities of local object-based computations (Steps 1 and 6) and computation on local integer arrays (Steps 2, 3 and 4) within a subcube are proportional to the number of local objects and the semiparameter (height plus width), respectively, of the rectangular subregion assigned to that subcube. Hence, the complexities of local computations within a subcube during the parallel ray tracing and parallel subdivision algorithms depend on the same factors: the number of local objects, and the height and width of the rectangular subregion assigned to that subcube. However, the dependence is multiplicative in parallel ray tracing, whereas it is additive in parallel subdivision. Hence, this deviation in the load balance measures of these two parallel algorithms may introduce a load imbalance among subcubes during parallel subdivision, since the proposed parallel subdivision algorithm inherently operates in accordance with the mapping strategy adopted by the recursive spatial bisection scheme, which tries to maintain a load balance during parallel ray tracing. This type of load imbalance is referred to here as intersubcube imbalance. There is no load imbalance among the processors of the individual subcubes during the local integer computation at Steps 2, 3 and 4, since each processor of a subcube operates on local integer arrays of the same size. However, processors of the same subcube may hold different numbers of local objects belonging to the respective subregion during a particular stage of the algorithm. This type of load imbalance, which is referred to here as intrasubcube imbalance, may introduce an imbalance during the concurrent objectbased computations (Steps 1 and 6) between the processors of the same subcube. An intrasubcube load imbalance may introduce processor idle time during both the global synchronization at Step 3 (global vector-sum operation) and object exchange synchronization at Step 6 within subcubes. The initial random distribution of objects to processors is an attempt to reduce intrasubcube load imbalances.

The communications overhead of the proposed parallel algorithm involves two components: the number and volume of communications. In a medium-to-coarse grain architecture with high communications latency, the number of communications may be a crucial factor in the performance of the parallel algorithm. Each one of the intrasubcube global operations at Steps 3 and 5 require $c+1$ concurrent exchange communication steps at stage $c$. Under perfect load balance conditions, these global communications within different subcubes will be performed concurrently. Hence, the total number of concurrent communications due to these intrasubcube global operations is $d(d+1)$. Thus, the total number of concurrent communications becomes $d(d+2)$ since the object exchange operations (Step 6) require $d$ concurrent communications in total under perfect load balance conditions. Hence, the percentage overhead due to the number of communications is negligible for sufficiently large granularity $(N / P)$ values.

The volume of concurrent communications during an individual intrasubcube global minimum operation (Step $5)$ is only $2(c+1)$ integers at stage $c$. On the other hand, the volume of the concurrent communication during an individual intrasubcube global vector-sum operation (Step 3) is $2(c+1)(n+m)$ integers, where $n+m$ denotes the semiperimeter of the rectangular subregion assigned to that subcube at stage $c$. That is, the total volume of this type of communications depend on the semiperimeter of the initial window and $d$. Hence, the percentage overhead 
due to these types of integer communications decreases with increasing scene complexity for a fixed window size. The total volume of communication due to the object migrations is a more crucial factor in the parallel performance of the proposed algorithm. Under averagecase conditions, half of the objects can be assumed to migrate at each stage of the algorithm. Hence, if shared objects are ignored, the total volume of communications due to object migrations can be assumed to be $(N / 2) \log _{2} P$ objects. Experiments on various scenes yield results that are very close to this average-case behaviour.

Under perfect load balance conditions, each processor is expected to hold $N / P$ objects and each processor pair can be assumed to exchange $N / 2 P$ objects, at each stage. Hence, under these conditions the total concurrent volume of communications due to object migrations will be $(N / 2 P) \log _{2} P$ objects. Experiments on various uniform scenes yield results that are very close to these expectations. However, results slightly deviate from these expectations for nonuniform scenes with objects clustered toward particular positions.

Figure 5 shows the efficiency curves for hypercubes of various dimensions as functions of the scene complexity. Efficiency values for a hypercube with $P$ processors are computed as $E_{P}=T_{1} / P T_{P}$, where $T_{1}$ and $T_{P}$ denote the execution times of the sequential and parallel subdivision programs on 1 and $P$ node processors, respectively. As seen in Figure 5, efficiency increases with increasing scene complexity for a fixed window resolution size. This increase can be attributed to two factors. The total number of communications stays fixed for a fixed hypercube size. Hence, the percentage overhead due to the total number of communications decreases with increasing scene complexity. Similarly, the volume of integer communications also stays fixed for fixed hypercube size and window resolution size. Hence, the percentage overhead due to the volume of integer communications also decreases with increasing scene complexity. As seen in Figure 5, efficiency values of close to $100 \%$ are obtained for $P=2$ processors since the initial even distribution of objects entirely avoids both intrasubcube and intersubcube load imbalances during

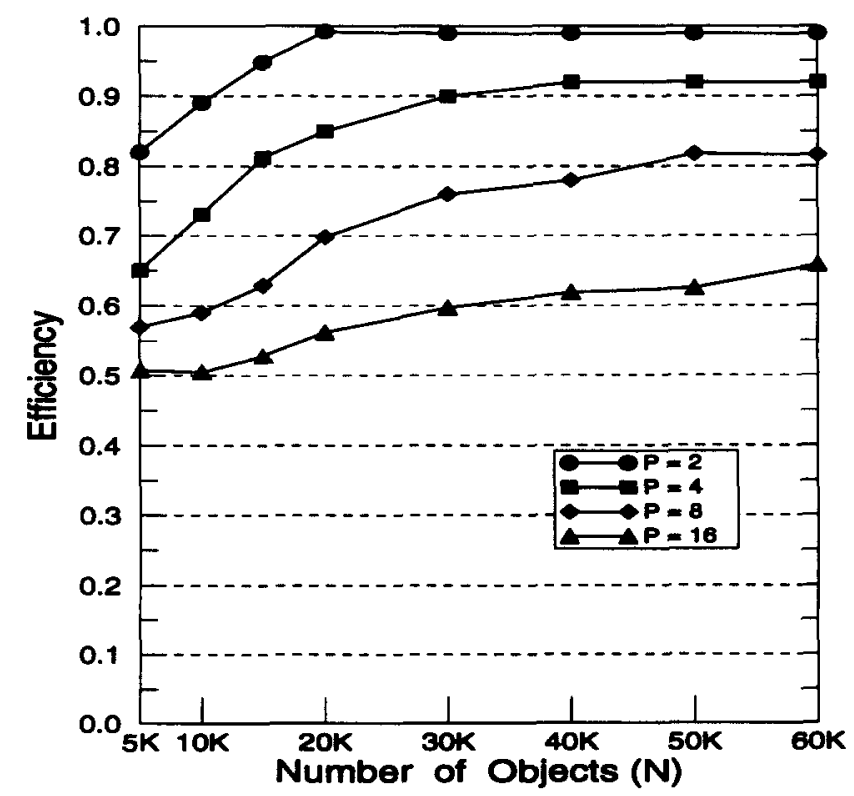

Figure 5 Efficiency curves for total number of objects in scene the first stage of the parallel BBSP algorithm. However, for a fixed scene instance, the efficiency decreases considerably with an increasing number of processors. This decrease is mainly due to the increase in the intersubcube load imbalances, since each doubling of the number of processors introduces an extra stage to the algorithm. Therefore, load rebalancing algorithms should be developed for larger numbers of processors.

\section{CONCLUSIONS}

An efficient subdivision algorithm based on BSP (called a BBSP algorithm) is proposed for object-based parallel ray tracing. The proposed BBSP algorithm tries to minimize the communications overhead during objectbased parallel ray tracing by exploiting data coherence. The other advantage of the proposed BBSP algorithm is that the subdivision process does not generate empty boxes. Empty boxes may occupy significantly large spaces. Besides, rays may take time skipping the empty boxes. The subdivision of space into $3 \mathrm{D}$ grid elements and in octree fashion is affected by these factors.

The preprocessing due to the subdivision of the $3 D$ space may be time consuming for complex scenes. An efficient parallel BBSP algorithm is proposed to reduce the preprocessing time. The implementation on an Intel iPSC/ 2 multicomputer has shown promising results.

\section{ACKNOWLEDGEMENTS}

The work described in this paper is partially supported by Intel Supercomputer Systems Division grant SSD100791-2, and Turkish Scientific and Technical Research Council (TÜBITAK) grant EEEAG-5.

\section{REFERENCES}

1 Green, $\mathbf{S} \mathbf{A}$ and Paddon, D J 'Exploiting coherence for multiprocessor ray tracing' Comput. Graph. \& Applic. Vol 9 No 6 (1989) pp 12-26

2 Goldsmith, J and Salmon, J 'Automatic creation of object hierarchies for ray tracing' Comput. Graph. \& Applic. Vol 7 No 3 (1987) pp 14-20

3 Glassner, A S 'Space subdivision for fast ray tracing' Comput. Graph. \& Applic. Vol 4 No 4 (1984) pp 15-22

4 Kaplan, $M$ R 'The use of spatial coherence in ray tracing' in Techniques for Computer Graphics Springer-Verlag (1987) pp 173-193

5 Isşler, V, Aykanat, C and Özgüç, B 'Subdivision of 3D space based on the graph partitioning for parallel ray tracing' Proc. 2nd Eurographics Wkshp. Rendering (1991)

6 Kobayashi, H, Nishimura, S, Kubota, H, Nakamura, T and Shigei, $Y$ 'Load balancing strategies for a parallel ray-tracing system based on constant subdivision' Visual Comput. Vol 4 (1988) pp 197-209

7 Priol, T and Bouatouch, K 'Static load balancing for a parallel ray tracing' Visual Comput. Vol 5 (1989) pp 109-119

8 Fujimoto, A, Tanaka, T and Iwata, K 'ARTS: accelerated ray-tracing system' Comput. Graph. \& Applic. Vol 5 No 2 (1985) pp 16-26

9 Fuchs, H, Kedem, Z M and Naylor, B F 'On visible surface generation by priori tree structures' Siggraph ' 80 Proc. Vol 14 No 3 (1980) pp 124-133

10 MacDonald, J D and Booth, K S 'Heuristics for ray tracing using space subdivision' Visual Comput. Vol 6 (1990) pp 153-166

1 Ozgüner, F and Aykanat, C'A reconfiguration algorithm for fault tolerance in a hypercube multiprocessor' Inf. Proc. Lett. Vol 29 (1988) pp 247-254 
Efficient parallel spatial subdivision algorithm for object-based parallel ray tracing: C Aykanat et al.

12 McNeill, M D J, Shah, B C, Hébert, M P, Lister P F and Grimsdale, R L 'Performance of space subdivision techniques in ray tracing' Comput. Graph. Forum Vol 11 No 4(1992) pp 213-220

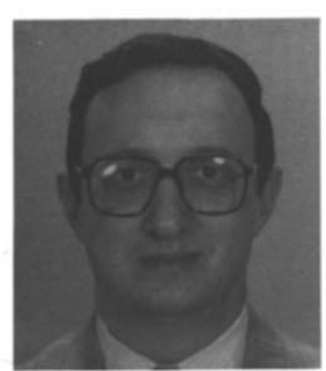

Bülent Özgüc joined the Bilkent University Faculty of Engineering, Turkey, in 1986. He is a professor of computer science and the dean of the Faculty of Art, Design and Architecture. He has taught at the University of Pennsylvania, USA, Philadelphia College of Arts, USA, and the Middle East Technical University, Turkey, and he worked as a member of the research staff at the Schlumberger Palo Alto Research Center. USA. For the last 15 years, he has been active in the field of computer graphics and animation. He received a BArch and an MArch in architecture from the Middle East Technical University in 1972 and 1973. He received an MS in architectural technology from Columbia University, USA, and a PhD in a joint program of architecture and computer graphics from the University of Pennsyluania in 1974 and 1978.

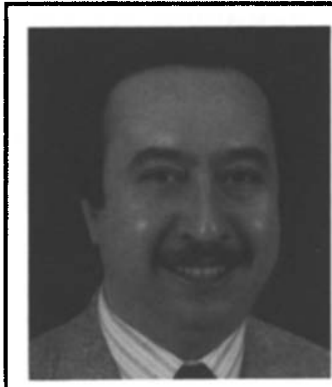

Cevdet Aykanat received a $B S$ and an MS from the Middle East Technical University, Turkey, and a PhD from Ohio State University, USA, all in electrical engineering. He was a Fulbright scholar during his PhD studies. He worked at the Intel Supercomputer Systems Division, USA, as a research associate. Since October 1988, he has been with the Department of Computer Engineering and Information Science, Bilkent University, Turkey, where he is an associate professor. His research interests include parallel computer architectures, parallel algorithms, parallel computer graphics applications, neural network algorithms, and fault-tolerant computing.

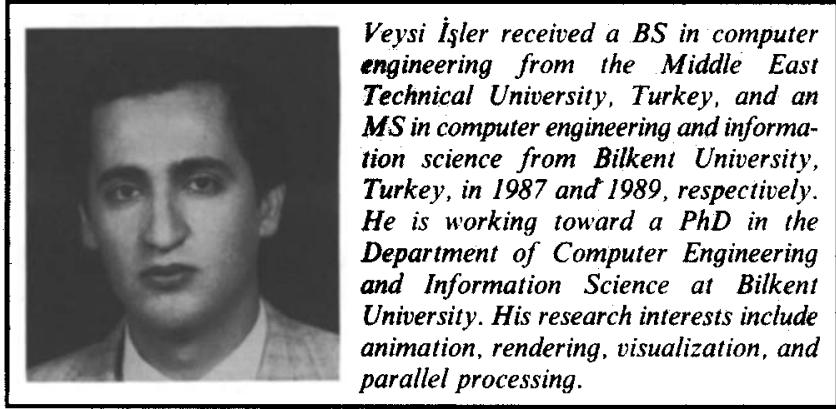

